\title{
Grid indentation analysis of mechanical properties of composite electrodes in Li-ion
}

\section{batteries}

\author{
Luize Scalco de Vasconcelos ${ }^{\text {a }}$, Rong Xu ${ }^{\text {a }}$, Jianlin $\mathrm{Li}^{\mathrm{b}}{ }^{\mathrm{b}}$, Kejie Zhao ${ }^{\mathrm{a}, *}$ \\ ${ }^{a}$ School of Mechanical Engineering, Purdue University, West Lafayette, IN 47906, USA \\ ${ }^{\mathrm{b}}$ Energy and Transportation Science Division, Oak Ridge National Laboratory, Oak Ridge, TN, \\ 37831-6479, USA
}

\begin{abstract}
Electrodes in commercial rechargeable batteries are microscopically heterogeneous materials. The constituent components, including active materials, polymeric binders, and porous conductive matrix, often have large variation in their mechanical properties, making the mechanical characterization of composite electrodes a challenging task. In a model system of $\mathrm{LiNi}_{0.5} \mathrm{Mn}_{0.3} \mathrm{Co}_{0.2} \mathrm{O}_{2}$ cathode, we employ the instrumented grid indentation to determine the elastic modulus and hardness of the constituent phases. The approach relies on a large array of nanoindentation experiments and statistical analysis of the resulting data provided that the maximum indentation depth is carefully chosen. The statistically extracted properties of the active particles and the surrounding medium are in good agreement with the tests of targeted indentation at selected sites. The combinatory technique of grid indentation and statistical deconvolution represents a fast and reliable route to quantify the mechanical properties of composite electrodes that feed the parametric input for the mechanics models.
\end{abstract}

Keywords: NMC cathode, grid indentation, statistical analysis, mechanical properties, batteries.

*Corresponding author: kjzhao@ purdue.edu_(K. Zhao) 


\section{Introduction}

Li-ion batteries play a central role in portable electronics and electric vehicles $[1,2]$. Technological improvements in rechargeable batteries are being driven by the ever-increasing demand on materials of long lifespan, high energy density, fast charging and power output, low cost, and safe operation [3]. Mechanical stability is an important measure of high-performance batteries. For instance, the drastic change in volume is an intrinsic drawback of high-capacity electrodes [4]. The volumetric strain is linearly proportional to the number of absorbed Li in alloying-type anodes and it reaches over $300 \%$ in Si which has the highest theoretical specific capacity [5]. As such, mechanical degradation places a roadblock in the implementation of highcapacity electrodes in commercial batteries. In high-power-output batteries of high current and deep discharge, structural disintegration impedes electron conduction and increases the ohmic and thermal resistance which can result in catastrophic failure of batteries [6]. More recently, mechanical stability becomes a major criterion in the selection of solid electrolyte for solid-state batteries. In addition to possessing appropriate electrochemical properties, polymer electrolyte must exhibit high mechanical strength in order to suppress the dendrite growth of Li metals and to enable large-scale manufacturing of the devices $[7,8]$.

The mechanics perspectives provide important insight in the optimum design of batteries. Numerous theoretical modeling and experiments have been conducted to characterize the large deformation, stress generation, and fracture in electrodes modulated by the electrochemical processes of lithiation and delithiation [9-27]. The mechanical properties of electrodes are prerequisite parameters for the mechanics models. Nevertheless, elastic modulus, hardness, and fracture strength of most emerging electrode materials are unknown a priori that makes theoretical predictions questionable. The difficulties underlying the experimental 
characterization on the mechanical properties of battery materials are twofold. First, electrodes in commercial batteries are heterogeneous materials consisting of metal- or ceramic-like active materials, polymeric binders, and porous carbon black (CB) conductive matrix [28]. The constituent components have large difference in their mechanical properties - the elastic modulus changes by 2-3 orders of magnitude for instance. In addition, the mechanical properties of composite electrodes are highly dependent on the packing density of the active material from the elementary powders and tortuosity of the surrounding medium [29]. Determining the mechanical properties of individual phases in the microscopically heterogeneous structures is a challenge. Second, the mechanical properties of active materials may vary significantly with the state of charge, for example, elastic modulus of graphite increases by three fold upon lithiation [30], and $\mathrm{Si}$ experiences a transition of brittle behavior in its pure form to a ductile material in the lithiated state [31-33]. Few data are available so far that compile the dependence of mechanical properties of electrodes on the state of charge.

We employ the technique of grid indentation that is suited to measure the mechanical properties of composite electrodes of high heterogeneity at the nano- to microscale [34, 35]. Grid indentation relies on a massive array of nanoindentation and statistical deconvolution of experimental data to extract the mechanical properties of individual components. Fig. 1 (a) sketches a material composed of two phases of distinct properties. Provided that the indentation depth is much smaller than the characteristic size of the two phases and the grid spacing is larger than the size of the indentation impression, a large number of indentations on the sample surface probe the mechanical properties of either phase with the probability that equals the surface fraction. Assuming that the distribution of the mechanical property of each phase can be described by the Gaussian distribution, grid indentation yields a multimodal probability function 
that allows determination of properties of each phase, Fig.1 (b). We apply grid indentation to a model system of $\mathrm{LiNi}_{0.5} \mathrm{Mn}_{0.3} \mathrm{Co}_{0.2} \mathrm{O}_{2}$ (NMC532) cathode for commercial batteries. $\mathrm{LiNi}_{x} \mathrm{Mn}_{y} \mathrm{Co}_{1-x-y} \mathrm{O}_{2}(\mathrm{NMC})$ is a class of cathode material attractive for the electric vehicle applications [36-39]. NMC is generally comprised of alternating Li and transition-metal layers where the composition of $\mathrm{Ni}, \mathrm{Mn}$, and Co can be tuned to optimize the performance in capacity, cyclic rate, electrochemical stability, and lifetime. The mechanical properties of the NMC electrodes have been widely unknown.

We validate grid indentation of the NMC532 electrode by comparing with the results of targeted indentation at selected sites. The maximum indentation depth is carefully chosen to avoid the effect of particle morphology at shallow indentation and the effect of surrounding medium at deep indentation. Grid indentation provides a fast and reliable route to characterize the mechanical properties of heterogeneous composite electrodes and will become a valuable tool in the design of resilient commercial batteries.

\section{Experimental procedure and statistical analysis}

\section{Sample preparation}

As-received $\mathrm{LiNi}_{0.5} \mathrm{Mn}_{0.3} \mathrm{Co}_{0.2} \mathrm{O}_{2}$ (NMC532, Toda America) powders, polyvinylidene fluoride, (PVDF, Solvay, 5130), carbon black (CB, Denka, powder grade), and Nmethylpyrrolidone (NMP, Sigma Aldrich) were used to prepare NMC cathodes by slot-die coating, which consist of $90 \mathrm{wt} \%$ NMC532, $5 \mathrm{wt} \%$ PVDF, and $5 \mathrm{wt} \%$ carbon black. Detailed fabrication method can be found in reference [40]. The areal loading of the NMC cathode was $12.5 \mathrm{mg} / \mathrm{cm}^{2}$. Samples composed of only CB and PVDF with 50/50 weight percentage were also prepared for the indentation test. 
Fig. 2 shows the scanning electron microscopy (SEM) images on the cathode microstructure consisting of nearly spherical NMC particles (estimated average diameter of approximately $10 \mu \mathrm{m}$ ) and a CB/PVDF porous matrix. Fig. 2 (b) shows the magnified view on a single NMC532 particle which is closely packed by primary powders. The electrode thickness (excluding the $\mathrm{Al}$ current collector) ranges from 45 to $57 \mu \mathrm{m}$ as shown in Fig. 2 (c).

The porosity $\varepsilon$ of the sample is calibrated using the following formula,

$$
\varepsilon=\frac{V_{m}-V_{t}}{V_{m}}=\frac{A_{s} h_{s}-\left(w_{s}-A_{s} h_{A l} \rho_{A l}\right)\left(\frac{f_{N M C}}{\rho_{N M C}}+\frac{f_{P V D F}}{\rho_{P V D F}}+\frac{f_{C B}}{\rho_{C B}}\right)}{A_{s} h_{s}}
$$

where the volume of the electrode (excluding the Al current collector) is $V_{m}=A_{s} h_{s}$, and the theoretical volume of zero porosity is $V_{t}$. $A_{s}$ is the surface area, $h_{A l}$ and $h_{s}$ are the thickness of the Al substrate and the electrode, respectively. $w_{s}$ represents the sample weight and $f$ the weight fraction of individual components. The theoretical density $(\rho)$ of NMC, CB, PVDF, and Al foil are, respectively, 4.77, 1.90, 1.76 and $2.70 \mathrm{mg} / \mathrm{mm}^{3}$. Table 1 lists the parameters used to calibrate the porosities of three samples of NMC electrode and CB/PVDF. The average porosities are $61 \%$ for CB/PVDF and $56 \%$ for NMC cathode.

Table 1. Porosity calibration of CB/PVDF and NMC cathode samples

\begin{tabular}{llllllll}
\hline \multirow{2}{*}{ Sample } & \multicolumn{2}{l}{ CB/PVDF } & & \multicolumn{3}{l}{ Cathode } \\
\cline { 2 - 4 } \cline { 7 - 8 } & 1 & 2 & 3 & & 1 & 2 & 3 \\
\hline$A_{s}\left[\mathrm{~mm}^{2}\right]$ & 145.81 & 712.09 & 174.85 & & 2886.65 & 1179.34 & 591.46 \\
$h_{s}[\mu \mathrm{m}]$ & 17.74 & 17.74 & 17.74 & & 56.93 & 55.33 & 57.26 \\
$h_{A l}[\mu \mathrm{m}]$ & 16.57 & 16.57 & 16.57 & & 0.015 & 0.015 & 0.015 \\
$w_{s}[\mathrm{mg}]$ & 1.47 & 6.67 & 7.49 & & 418.64 & 166.13 & 83.60 \\
$\varepsilon[\%]$ & 61.39 & 63.71 & 57.95 & & 55.32 & 55.86 & 57.15 \\
\hline
\end{tabular}

As-prepared samples were not calendered. We polished the sample surface for the indentation experiments. Samples were adhered to a glass using Crystalbond, and polished 
successively using $3 \mu \mathrm{m}$ and $1 \mu \mathrm{m}$ diamond paste with microid diamond extender and $0.05 \mu \mathrm{m}$ colloidal silica suspension.

\section{Grid indentation and targeted indentation}

Instrumented indentation (Keysight G200) was employed to measure the mechanical properties of the electrode. Indentation tests were performed using Berkovich tip at a constant strain rate of $0.05 \mathrm{~s}^{-1}$. Poisson ratio of 0.3 and $5 \mathrm{~s}$ peak hold time were used.

A common approach to obtain the properties of single phases in a heterogeneous material is targeted indentation at the desired phase only. This process requires careful selection of the indentation location and examination to ensure that results are not affected by the surrounding medium [41]. A faster and more practical alternative is to use grid indentation technique followed by statistical deconvolution. Both methods are used and results are compared in this work.

In targeted indentation, the mechanical properties of NMC particles are determined by indenting the center of selected particles of diameter larger than $8 \mu \mathrm{m}$. The distance between the impression locations and the adjacent CB/PVDF phase is large enough to avoid the effect of the surrounding medium. The indentation sites can be specified precisely as a result of the fine polishing that provide a flat and smooth surface. The targeted indentation approach, however, is not effective to obtain the properties of the CB/PVDF matrix because NMC particles are likely to be buried underneath the CB/PVDF coating. Even relatively shallow indents may probe NMC particles immediately below the indented area. As a result, we perform standard indentation on CB/PVDF samples to obtain the mechanical properties of the porous matrix.

Grid indentation is suited to measure the mechanical properties of heterogeneous materials. The technique relies on a large array of nanoindentation. In our tests, the indentation 
depth (typically $100 \mathrm{~nm}$ ) is much smaller than the characteristic size of the constituent phases, and the spacing between the indents ( 3 to $5 \mu \mathrm{m}$ ) is larger than the size of individual impression. Given that the constituent components possess distinct mechanical properties, grid indentation yields a multimodal probability function that allows determination of each phase using statistical analysis described as follows.

\section{Statistical analysis}

Let $x$ be the measured mechanical property - in this case, it is either elastic modulus $(E)$ or hardness $(H)$. Assuming that the mechanical property of each phase can be best described as Gaussian distribution functions, the theoretical cumulative distribution function $C_{i}(\mathrm{CDF})$ of the $i$-th phase is given by

$$
C_{i}(x)=\int_{0}^{x} \frac{1}{\sigma_{i} \sqrt{2 \pi}} e^{-\frac{\left(u-\mu_{i}\right)^{2}}{2 \sigma_{i}^{2}}} d u
$$

where $\mu_{i}$ is the mean value and $\sigma_{i}$ the standard deviation .

Let $f_{i}$ be the normalized surface fraction of the $i$-th phase with the condition $\sum_{i=1}^{n} f_{i}=1, n$ the total number of phases and $C_{\text {exp }}$ the normalized cumulative distribution of the experimental data. The unknowns $\left\{f_{i}, \mu_{i}^{E}, \sigma_{i}^{E}, \mu_{i}^{H}, \sigma_{i}^{H}\right\}$ are determined by minimizing the difference between the experimental CDFs and the weighted modal-phase CDFs while maintaining the same surface fraction $f_{i}$ in the elastic modulus and hardness CDFs,

$$
\min \left[\left(\sum_{i=1}^{n} f_{i} C_{i}^{E}-C_{e x p}^{E}\right)^{2}+\left(\sum_{i=1}^{n} f_{i} C_{i}^{H}-C_{e x p}^{H}\right)^{2}\right]
$$

Here $C_{e x p}^{E}$ and $C_{e x p}^{H}$ correspond to the experimental cumulative distributions for elastic modulus and hardness, respectively. The results of statistical deconvolution are estimates of the mean and 
standard deviation of elastic modulus and hardness of each phase, and surface fraction. The surface fraction of individual components in the selected indentation area may deviate from the overall volume fraction in the samples.

\section{Results and discussion}

We start with targeted indentation at selected sites on NMC particles and CB/PVDF matrix to quantify the dependence of measured properties on the maximum indentation depth. The test regions are chosen under optical imaging, Fig. 3 (a), and the indenter coordinates are recalibrated in each test to ensure that impression is performed at the specified locations. The inset figure in Fig. 3 (a) shows a typical force-displacement curve of nanoindentation with a set of $400 \mathrm{~nm}$ maximum displacement. Elastic modulus and hardness are obtained following the Oliver-Pharr method [42]. Fig. 3 (b) shows the histograms of elastic modulus and hardness at the maximum indentation depth of 50, 100, and $150 \mathrm{~nm}$, respectively. All the data obey a typical normal distribution with relatively small deviation, which indicates that the indentations are indeed performed within the particles. The measured mechanical properties of NMC particles are sensitive to the maximum indentation depth due to the effect of particle microstructure (shown in Fig. 2 (b)) at shallow indentation and the effect of surrounding CB/PVDF medium at deep indentation. Fig. 3 (c) shows the dependence of measured elastic modulus and hardness on the maximum indentation depth in the range of $50 \mathrm{~nm}$ to $420 \mathrm{~nm}$. The red spots are the mean and error bars are the standard deviations. The range marked in the blue rectangles represents the optimum indentation depth to measure the intrinsic properties of NMC particles. Results below $75 \mathrm{~nm}$ are likely to be influenced by the surface features of the particles and also by the instrument precision limitation. At the other end, indentation performed over $150 \mathrm{~nm}$ might be affected by the surrounding compliant medium as well as particle internal porosity, resulting in a 
gradually decreasing modulus and hardness at larger indentation depth. The average values of Young's modulus and hardness are $138.73 \mathrm{GPa}$ and $8.89 \mathrm{GPa}$, respectively, in the optimum window of indentation depth ranging from $75 \mathrm{~nm}$ to $150 \mathrm{~nm}$.

Similar targeted indentations are performed on CB/PVDF samples to evaluate the mechanical properties at various indentation depths. A total of 350 tests are carried out at different sites. The maximum indentation depth should be chosen to avoid the effect of surface roughness at shallow indentation and the effect of Al substrate at deep indentation. Fig. 4 shows the dependence of measured Young's modulus and hardness on the indentation depth in the range of $200 \mathrm{~nm}$ to $2200 \mathrm{~nm}$. The mechanical properties are relatively insensitive to the indentation depth and the average values of Young's modulus and hardness are $1.78 \mathrm{GPa}$ and $0.043 \mathrm{GPa}$, respectively. It is worth noting that the mechanical properties of the CB/PVDF samples are highly dependent on the porosity - the tested samples have a porosity of $61 \%$ which is close to the typical set in commercial batteries. One may expect to obtain higher values of modulus and hardness for samples of low porosity.

We next use the technique of grid indentation to determine the mechanical properties of individual components in the composite electrodes. We regard the NMC particle as one constituent phase and the mixture of surrounding CB/PVDF as another general component. In grid indentation, we fix the maximum indentation depth as $100 \mathrm{~nm}$. Fig. 5 shows the distribution maps of Young's modulus and hardness generated from 121 tests in a $33 \mu \mathrm{m} \times 33 \mu \mathrm{m}$ area. An excellent match between the mechanical properties and the phase distribution in the optical image (Fig. 5 (a)) is clearly seen - higher values are shown on NMC particles (stiff and hard) and lower values in the CB/PVDF matrix (compliant and soft). In addition, due to the small size of the particles embedded in the matrix, a significant fraction of the data exhibit mixed properties 
of the particles and the matrix. Such mixed regions are referred as interface, the third constituent phase of the composite electrode in the light of the mechanical properties. The interface would exhibit a large variation in elastic modulus and hardness within the limits of the properties of NMC particles and CB/PVDF matrix. In Fig. 5 (b) and (c), we can estimate the range of the mechanical properties for each phase, which can be used to facilitate the optimization algorithm in statistical analysis. For example, the contours shows that the modulus of the particles are in between 120 and $160 \mathrm{GPa}$, while the modulus of the matrix lies within 0.1 and $10 \mathrm{GPa}$. Such information can be used to distinguish the individual peaks in the probability distributions.

The results of grid indentation are analyzed using the technique of statistical deconvolution to extract the mechanical properties of the NMC particles, CB/PVDF matrix, and the interface. The principle is described in Eq. (3). In the numerical fitting, a robust optimization algorithm requires minimum input from the user, and is able to consistently converge to the global minimum as opposed to a local minimum. The genetic algorithm is set to look for the best parameters throughout 150 iterations and 3000 points in each iteration. This way, the optimization is insensitive to the initial guesses and consistently converges to similar results. Fig. 6 (a) and (c) show the fitting of experimental CDFs of elastic modulus and hardness using trimodal Gaussian distributions, (b) and (d) show the experimental probability distribution functions (PDFs) along with the numerical PDFs using the same set of parameters in the CDFs fitting. It is noteworthy that the PDFs fit well to the experimental distributions even the parameters are completely determined by minimizing the CDFs. Table 2 summarizes the surface fractions, mean and standard deviation of elastic modulus and hardness obtained from the statistical analysis, and comparison with the results of targeted indentations. The mechanical properties of the NMC particles determined by grid indentation and targeted indentation are in 
good agreement. This may be understood that the NMC particles are stiff and hard materials and the experimental measurements are minimally influenced by the surrounding medium. For the $\mathrm{CB} / \mathrm{PVDF}$ matrix, grid indentation yields larger values of modulus and hardness by a factor of 2 3. This difference is mostly due to the fact that NMC particles are likely buried underneath the surface of CB/PVDF coating and even shallow indentation may probe the NMC particles in a portion of grid indentation. This comparison may not be unreasonable given the complexity of the microstructure of the composite samples.

Table 2. Surface fractions, elastic modulus, and hardness of individual components determined by grid indentation and targeted indentation.

\begin{tabular}{|c|c|c|c|c|c|}
\hline \multirow{2}{*}{$\begin{array}{l}\text { Material } \\
\text { Phase }\end{array}$} & \multirow{2}{*}{$\begin{array}{l}\text { Surface fraction [\%] } \\
\text { Grid Ind. }\end{array}$} & \multicolumn{2}{|c|}{ Modulus [GPa] $(\mu \pm \sigma)$} & \multicolumn{2}{|c|}{ Hardness [GPa] $(\mu \pm \sigma)$} \\
\hline & & Grid Ind. & Targeted Ind. & Grid Ind. & Targeted Ind. \\
\hline Particles & 38.40 & $123.02 \pm 20$ & $138.73 \pm 18.78$ & $7.78 \pm 1.40$ & $8.89 \pm 1.86$ \\
\hline Matrix & 15.64 & $4.40 \pm 2$ & $1.78 \pm 0.35$ & $0.13 \pm 0.01$ & $0.043 \pm 0.01$ \\
\hline Interface & 45.96 & $45.24 \pm 27.58$ & & $1.44 \pm 1.42$ & \\
\hline
\end{tabular}

\section{Conclusion}

We employ instrumented grid indentation to measure the mechanical properties of individual constituent components in a NMC cathode of high heterogeneity at the microscale. The approach applies a large array of nanoindentation and statistical analysis of the probability functions of the resulting data. The extracted elastic modulus and hardness of the NMC particles and the surrounding $\mathrm{CB} / \mathrm{PVDF}$ matrix are in good agreement with the tests of targeted indentation. The combinatory technique of grid indentation and statistical deconvolution provides a fast and practical route to determine the mechanical properties of heterogeneous materials that will feed the constitutive models of composite electrodes. We will investigate the evolution of the mechanical properties of electrodes in the course of electrochemical cycles in a future study. Grid indentation serves as a valuable tool in the characterization of mechanical 
behaviors of commercial electrodes as well as in design of high-performance rechargeable batteries.

\section{Acknowledgements}

The research project is supported by the start-up funds at Purdue University. K. Z. is grateful for the research funds of 3M nontenured faculty award. Research at Oak Ridge National Laboratory (ORNL), managed by UT Battelle, LLC, for the U.S. Department of Energy (DOE) under contract DE-AC05-00OR22725, utilized the DOE Battery Manufacturing R\&D Facility at ORNL sponsored by the Office of Energy Efficiency and Renewable Energy's Vehicle Technologies Office to fabricate electrode samples. 


\section{References}

1. Armand M, Tarascon JM. Building better batteries. Nature. 2008;451:652-657.

2. M.S. Whittingham, Materials challenges facing electrical energy storage, MRS Bulletin. 2008;33:411-419.

3. Tarascon JM, Armand M. Issues and challenges facing rechargeable lithium batteries. Nature. 2001;414:359-367.

4. Mukhopadhyay A, Sheldon BW. Deformation and stress in electrode materials for Li-ion batteries. Progress in Materials Science. 2014;63:58-116.

5. Obrovac M, Chevrier V. Alloy negative electrodes for Li-ion batteries. Chemistry Reviews. 2014;114:11444-11502.

6. Ning G, Haran B, Popov BN. Capacity fade study of lithium-ion batteries cycled at high discharge rates. Journal of Power Sources. 2003;117:160-169.

7. Yu S, Schmidt RD, Mendez RG, Herbert E, Dudney NJ, Wolfenstine JB, et al. Elastic properties of the solid electrolyte $\mathrm{Li}_{7} \mathrm{La}_{3} \mathrm{Zr}_{2} \mathrm{O}_{12}$ (LLZO). Chemistry of Materials. 2016;28:197-206.

8. Wang Y, Zhong WH. Development of electrolytes towards achieving safe and highperformance energy-storage devices: A review. ChemElectroChem. 2015;2:22-36.

9. Christensen J, Newman J. Stress generation and fracture in lithium insertion materials. Journal of the Solid State Electrochemistry. 2006;10:293-319.

10. Cheng YT, Verbrugge MW. Diffusion-induced stress, interfacial charge transfer, and criteria for avoiding crack initiation of electrode particles. Journal of the Electrochemical Society. 2010;157:A508-A516.

11. Haftbaradaran H, Xiao XC, Verbrugge MW, Gao HJ. Method to deduce the critical size for interfacial delamination of patterned electrode structures and application to lithiation of thin-film silicon islands. Journal of Power Sources. 2012;206:357-366.

12. Bower AF, Guduru PR, Sethuraman VA. A finite strain model of stress, diffusion, plastic flow, and electrochemical reactions in a lithium-ion half-cell. Journal of the Mechanics and Physics of Solids. 2011;59:804-828.

13. Cui ZW, Gao F, Qu JM. A finite deformation stress-dependent chemical potential and its applications to lithium ion batteries. Journal of the Mechanics and Physics of Solids. 2012;60:1280-1295.

14. Zhao KJ, Pharr M, Cai SQ, Vlassak JJ, Suo ZG. Large plastic deformation in highcapacity lithium-ion batteries caused by charge and discharge. Journal of the American Ceramic Society. 2011;94:S226-S235.

15. Yang H, Fan F, Liang W, Guo X, Zhu T, Zhang S. A chemo-mechanical model of lithiation in silicon. Journal of the Mechanics and Physics of Solids. 2014;70:349-361.

16. Huang X, Yang H, Liang W, Raju M, Terrones M, Crespi VH, et al. Lithiation induced corrosive fracture in defective carbon nanotubes. Applied Physics Letters. 2013; 103:153901.

17. Zhang $\mathrm{Y}$, Li $\mathrm{Y}$, Wang $\mathrm{Z}$, Zhao $\mathrm{K}$. Lithiation of $\mathrm{SiO}_{2}$ in Li-Ion batteries: In situ transmission electron microscopy experiments and theoretical studies. Nano letters. 2014;14:7161-7170.

18. Brassart L, Zhao K, Suo Z. Cyclic plasticity and shakedown in high-capacity electrodes of lithium-ion batteries. International Journal of Solids and Structures. 2013;50:11201129. 
19. Zhao KJ, Pharr M, Wan Q, Wang WL, Kaxiras E, Vlassak JJ, et al. Concurrent reaction and plasticity during initial lithiation of crystalline silicon in lithium-ion batteries. Journal of the Electrochemical Society. 2012;159:A238-A243.

20. Pharr M, Zhao KJ, Wang XW, Suo ZG, Vlassak JJ. Kinetics of initial lithiation of crystalline silicon electrodes of lithium-Ion batteries. Nano Letters. 2012;12:5039-5047.

21. Wang X, Yang A, Xia S. Fracture toughness characterization of lithiated germanium as an anode material for lithium-ion batteries. Journal of the Electrochemical Society. 2016;163:A90-A95.

22. Sethuraman VA, Chon MJ, Shimshak M, Srinivasan V, Guduru PR. In situ measurements of stress evolution in silicon thin films during electrochemical lithiation and delithiation. Journal of Power Sources. 2010;195:5062-5066.

23. Nadimpalli SP, Sethuraman VA, Abraham DP, Bower AF, Guduru PR. Stress evolution in lithium-ion composite electrodes during electrochemical cycling and resulting internal pressures on the cell casing. Journal of the Electrochemical Society. 2015;162:A2656A2563.

24. Yang H, Liang W, Guo X, Wang C-M, Zhang S. Strong kinetics-stress coupling in lithiation of Si and Ge anodes. Extreme Mechanics Letters. 2015;2:1-6.

25. Xu R, Zhao K. Mechanical interactions regulated kinetics and morphology of composite electrodes in Li-ion batteries. Extreme Mechanics Letters. 2015. doi:10.1016/j.eml.2015.10.004.

26. Klinsmann M, Rosato D, Kamlah M, McMeeking RM. Modeling crack growth during Li extraction in storage particles using a fracture phase field approach. Journal of the Electrochemical Society. 2016;163:A102-A218.

27. Kim S, Choi SJ, Zhao K, Yang H, Gobbi G, Zhang S, et al. Electrochemically driven mechanical energy harvesting. Nature communications. 2016;7:10146.

28. Zhao KJ, Pharr M, Vlassak JJ, Suo ZG. Fracture of electrodes in lithium-ion batteries caused by fast charging. Journal of Applied Physics. 2010;108:073517.

29. Vijayaraghavan B, Ely DR, Chiang Y-M, García-García R, García RE. An analytical method to determine tortuosity in rechargeable battery electrodes. Journal of the Electrochemical Society. 2012;159:A548-A552.

30. Qi Y, Guo H, Hector LG, Timmons A. Threefold increase in the Young's modulus of graphite negative electrode during lithium intercalation. Journal of the Electrochemical Society. 2010;157:A558-A566.

31. Zhao KJ, Wang WL, Gregoire J, Pharr M, Suo ZG, Vlassak JJ, et al. Lithium-assisted plastic deformation of silicon electrodes in lithium-Ion batteries: A first-principles theoretical study. Nano Letters. 2011;11:2962-2967.

32. Wang H, Wang X, Xia S, Chew HB. Brittle-to-ductile transition of lithiated silicon electrodes: Crazing to stable nanopore growth. The Journal of chemical physics. 2015;143:104703.

33. Ding B, Li X, Zhang X, Wu H, Xu Z, Gao H. Brittle versus ductile fracture mechanism transition in amorphous lithiated silicon: From intrinsic nanoscale cavitation to shear banding. Nano Energy. 2015;18:89-96.

34. Constantinides G, Chandran KR, Ulm F-J, Van Vliet K. Grid indentation analysis of composite microstructure and mechanics: principles and validation. Materials Science and Engineering: A. 2006;430:189-202. 
35. Ulm FJ, Vandamme M, Bobko C, Alberto Ortega J, Tai K, Ortiz C. Statistical indentation techniques for hydrated nanocomposites: concrete, bone, and shale. Journal of the American Ceramic Society. 2007;90:2677-2692.

36. Ohzuku T, Makimura Y. Layered lithium insertion material of $\mathrm{LiNi}_{1 / 2} \mathrm{Mn}_{1 / 2} \mathrm{O}_{2}$ : A possible alternative to $\mathrm{LiCoO}_{2}$ for advanced lithium-ion batteries. Chemistry Letters. 2001:744-745.

37. Lu Z, MacNeil D, Dahn J. Layered Li[ $\left.\mathrm{Ni}_{x} \mathrm{Co}_{1-2 \mathrm{x}} \mathrm{Mn}_{\mathrm{x}}\right] \mathrm{O}_{2}$ cathode materials for lithiumion batteries. Electrochemical and Solid-State Letters. 2001;4:A200-A203.

38. Mohanty D, Kalnaus S, Meisner RA, Rhodes KJ, Li J, Payzant EA, et al. Structural transformation of a lithium-rich $\mathrm{Li}_{1.2} \mathrm{Co}_{0.1} \mathrm{Mn}_{0.55} \mathrm{Ni}_{0.15} \mathrm{O}_{2}$ cathode during high voltage cycling resolved by in situ X-ray diffraction. Journal of Power Sources. 2013;229:239248.

39. Mohanty D, Li J, Nagpure SC, Wood DL, Daniel C. Understanding the structure and structural degradation mechanisms in high-voltage, lithium-manganese-rich lithium-ion battery cathode oxides: A review of materials diagnostics. MRS Energy \& Sustainability. 2015;2:E15.

40. Li J, Daniel C, An SJ, Wood D. Evaluation residual moisture in lithium-ion battery electrodes and its effect on electrode performance. MRS Advances. 2015:1-7

41. Nohava J, Haušild P, Houdková Š, Enžl R. Comparison of isolated indentation and grid indentation methods for HVOF sprayed cermets. Journal of Thermal Spray Technology. 2012;21:651-658.

42. Oliver WC, Pharr GM. An improved technique for determining hardness and elastic modulus using load and displacement sensing indentation experiments. Journal of Materials Research. 1992;7:1564-1583. 
(a)

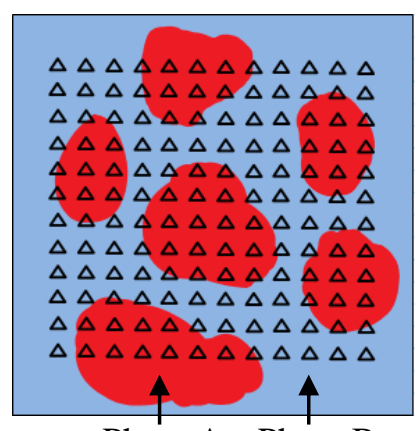

Phase A Phase B (b)

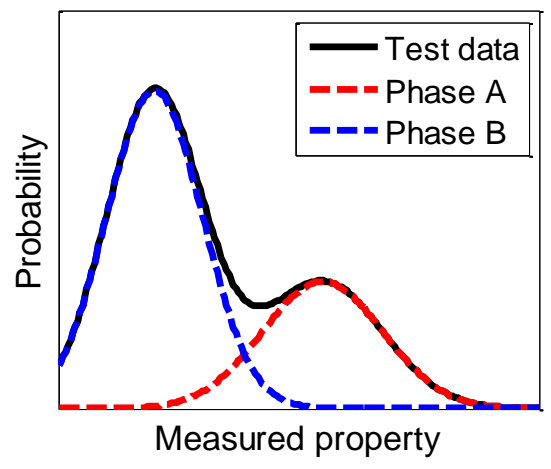

Fig. 1 (a) Schematic of grid indentation on a heterogeneous material. The red and blue colors represent different phases, and the triangles represent individual indentation sites. The indentation size is much smaller than the characteristic size of the phases and the grid spacing is larger than the size of indentation impression. (b) Grid indentation yields a multimodal probability function that allows determination of mechanical properties of the constituent phases. 
(a)

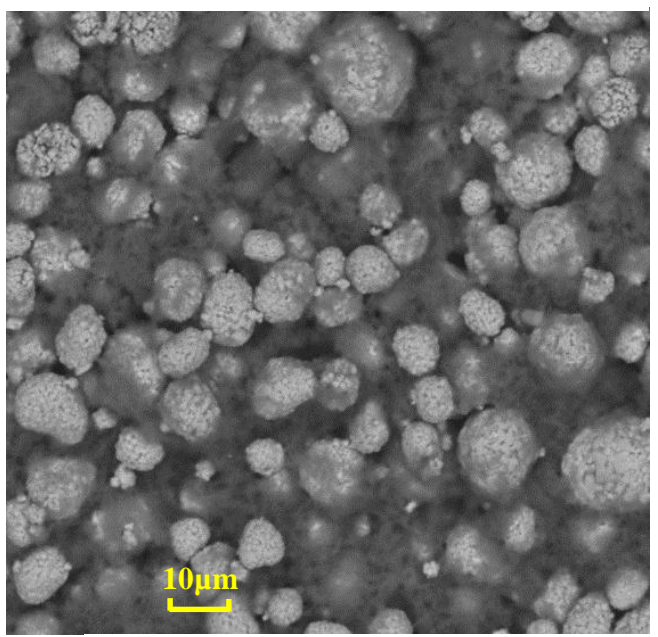

(c) (b)
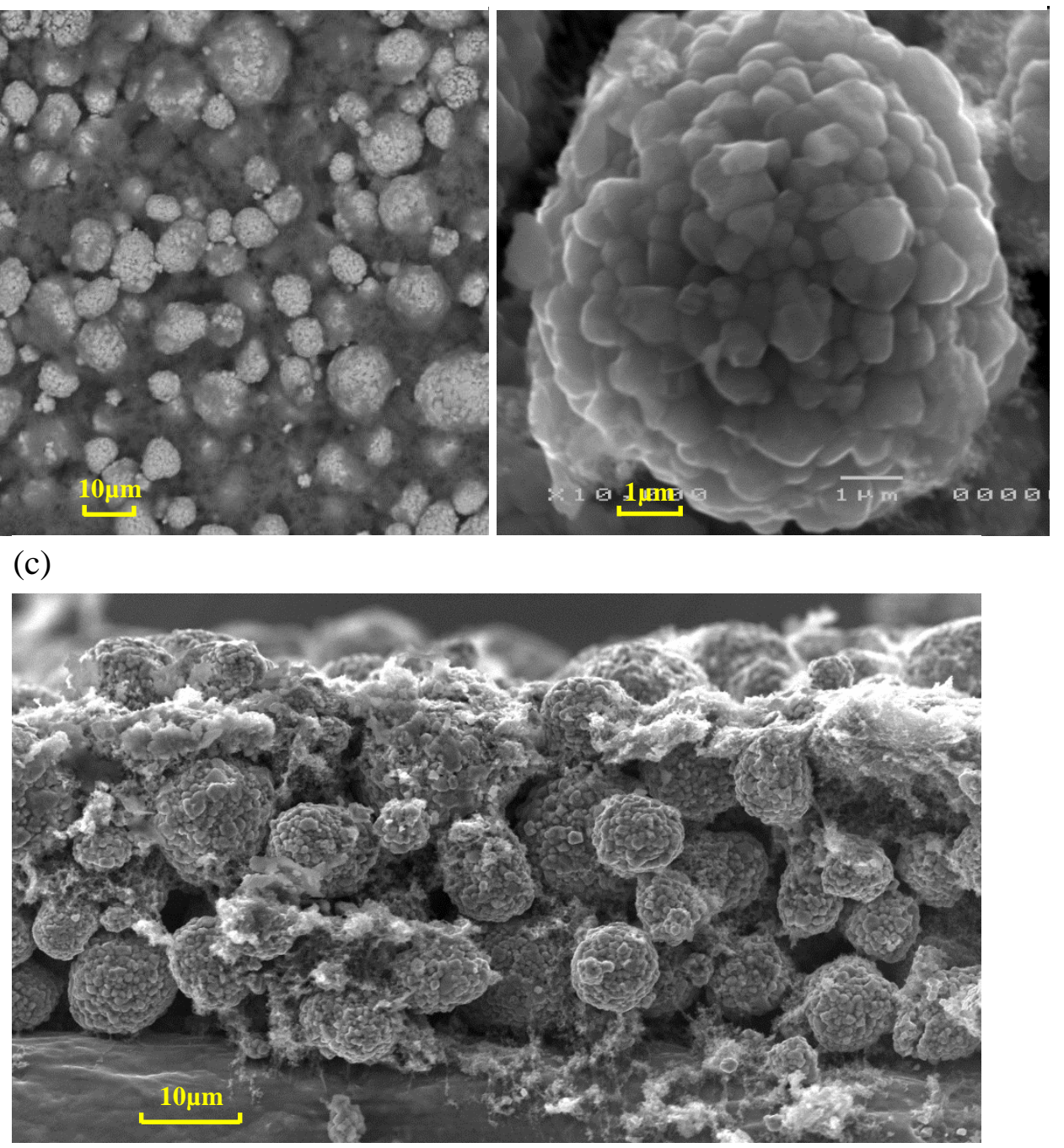

Fig. 2. SEM images of the cathode electrode composed of NMC532 particles, PVDF binders, and porous carbon black matrix. (a) Top view. (b) Magnified view on a single NMC532 particle. (c) Cross-section view. 
(a)

(b)
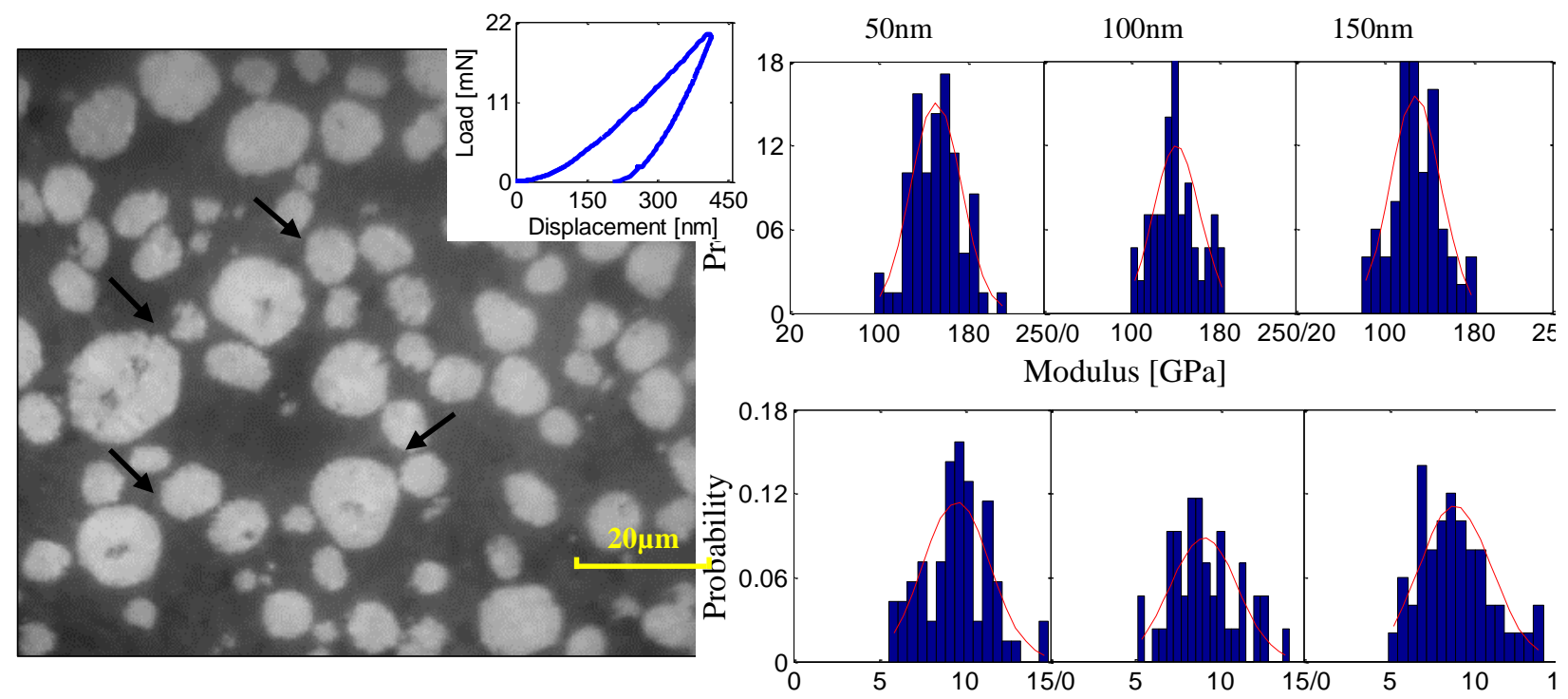

(c)
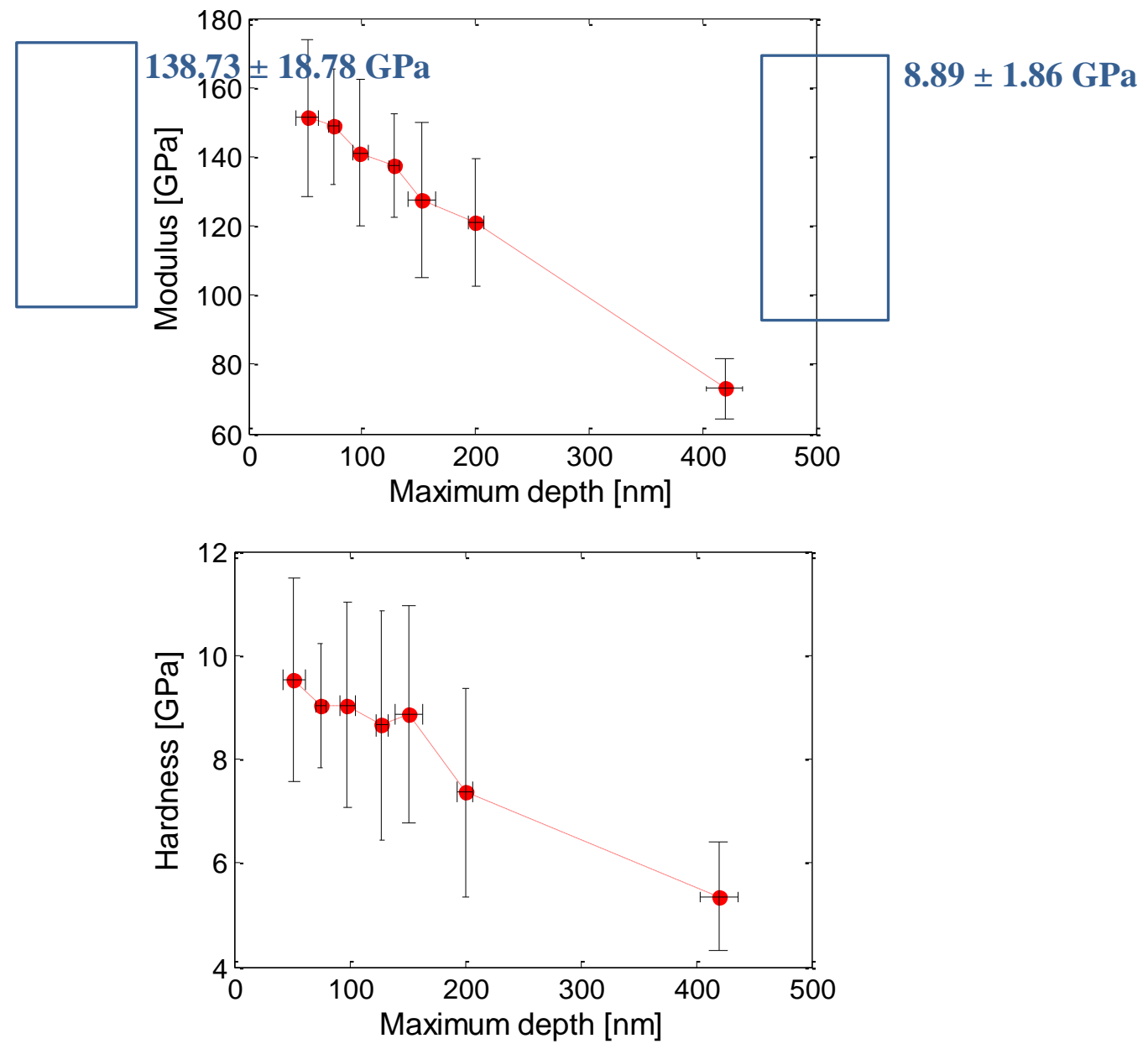
Fig. 3. (a) Targeted indentation on single NMC particles. The arrows point to the selected particles under optical imaging. The inset figure shows the typical load-displacement curve of nanoindentation. (b) Indentation histograms of Young's modulus and hardness at various indentation depths. (c) Dependence of Young's modulus and hardness on the maximum indentation depth. The blue rectangles mark the range in which the measured properties are less sensitive to the effect of particle microstructure at shallow indentation and the effect of surrounding medium at deep indentation. 
(a)
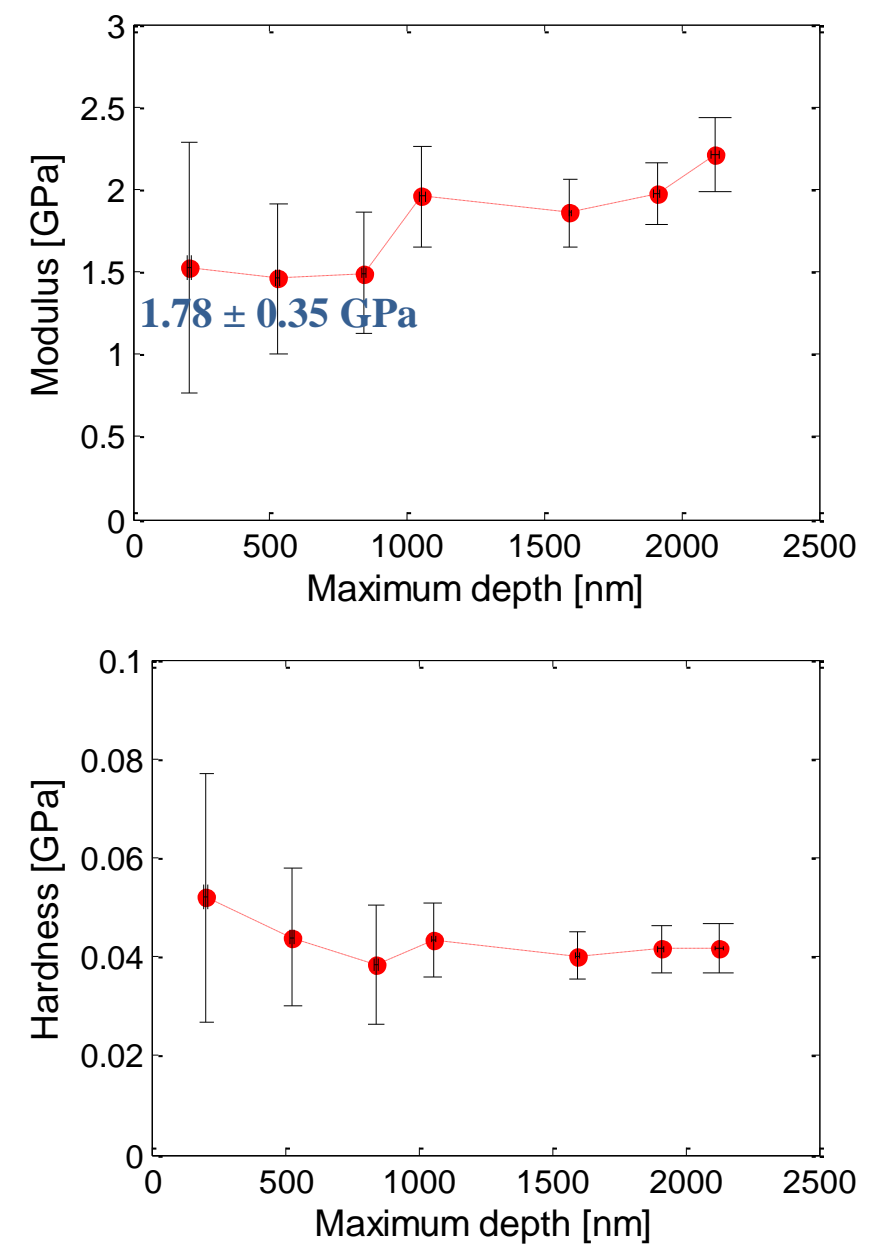

$0.043 \pm 0.01$ GPa

Fig. 4. (a) Young's modulus and (b) hardness of CB/PVDF sample measured at various indentation depths. The mechanical properties are relatively insensitive to the effect of surface roughness at shallow indentation and the substrate effect at deep indentation. 
(a)

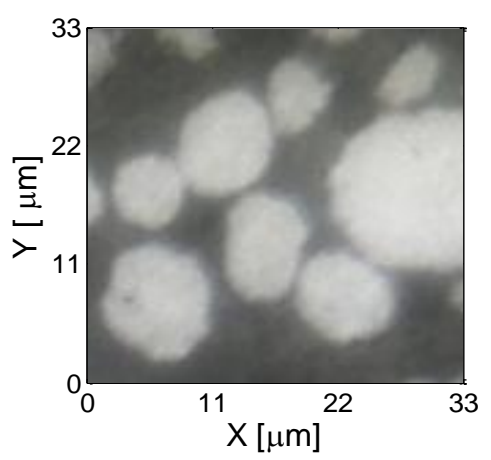

(b)

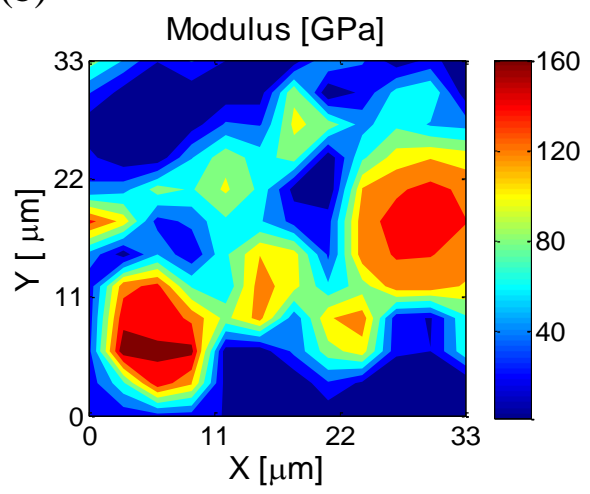

(c)

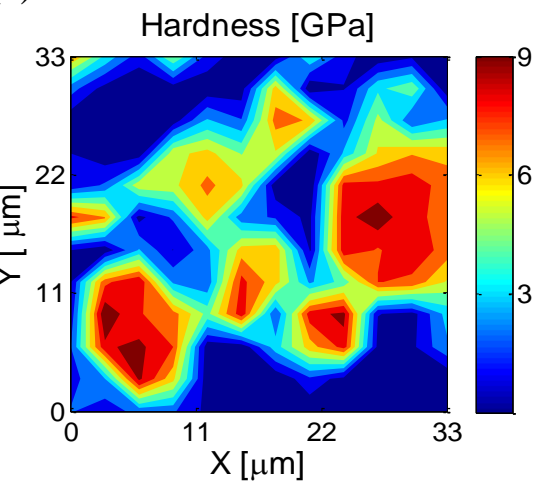

Fig. 5. (a) Optical image of a $33 \mu \mathrm{m} \times 33 \mu \mathrm{m}$ area for grid indentation. Contour plot of (b) Young's modulus and (c) hardness in the selected area. 

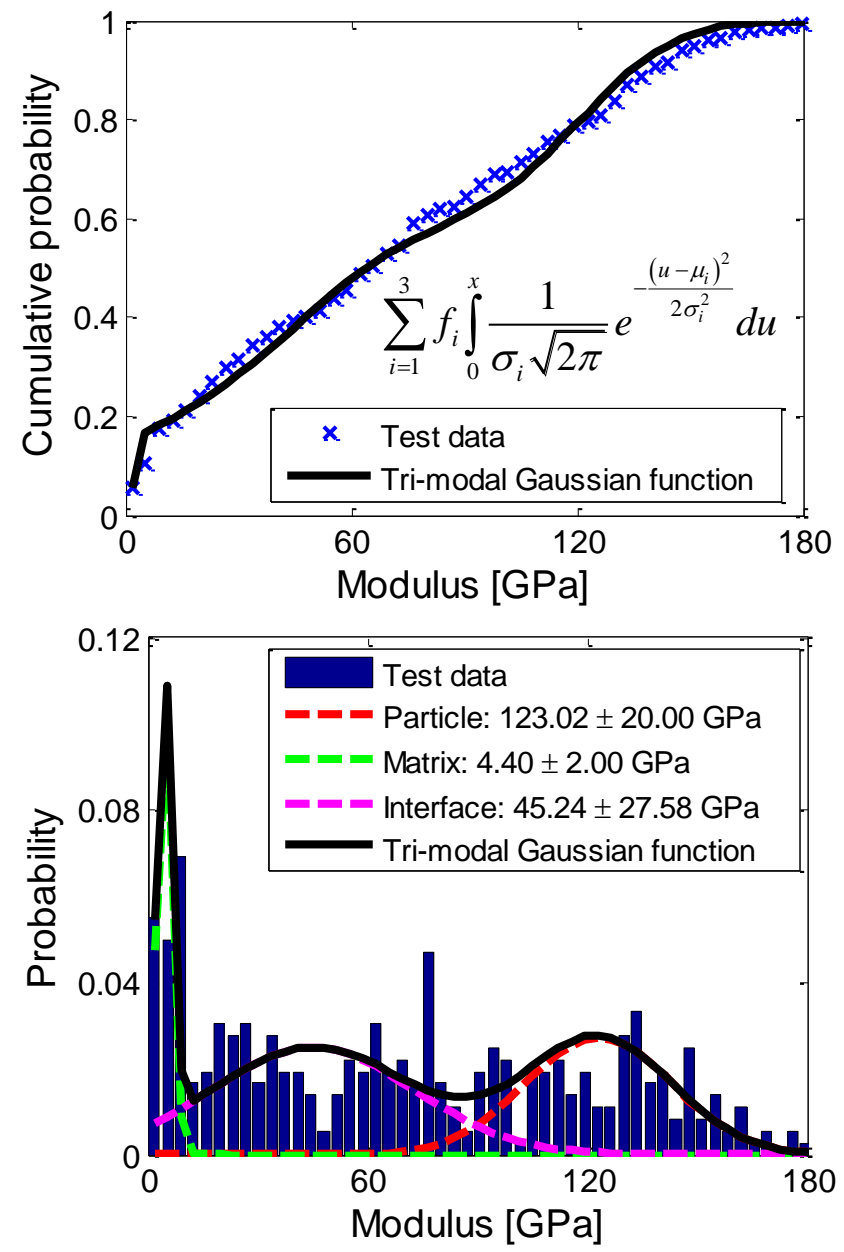

(c)

(d) 

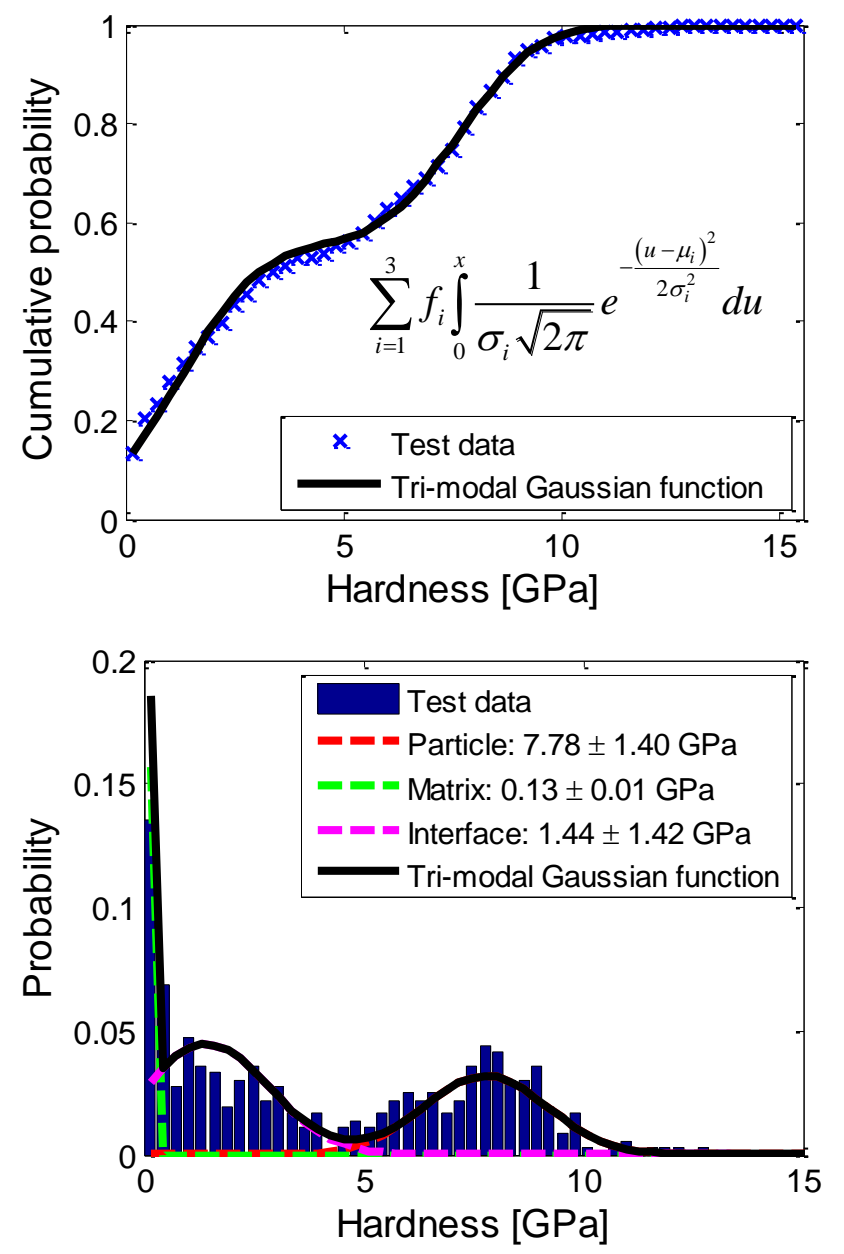

Fig. 6. (a) Cumulative probability of Young's modulus and tri-modal Gaussian fitting. (b) Plots of probability distribution function using the same set of parameters in (a). (c) Cumulative probability of hardness and tri-modal Gaussian fitting. (d) Plots of probability distribution function using the same set of parameters in (c). 